\title{
Associação de Modificação do Comportamento: contingências para a institucionalização da Análise do Comportamento no Brasil
}

\section{Associação de Modificação do Comportamento: contingencies for Behavior Analysis institutionalization in Brazil}

\section{Associação de Modificação do Comportamento: contingencias para la institucionalización de Análisis de la Conducta en Brasil}

\author{
Jaqueline de Andrade Torres', Gabriel Vieira Cândido², Rodrigo Lopes Miranda ${ }^{3}$
}

[1] Universidade Católica Dom Bosco (UCDB) [2] Universidade Federal de São Carlos (UFSCar) [3] Universidade Católica Dom Bosco (UCDB) I Título abreviado: Associação de Modificação do Comportamento | Endereço para correspondência: Universidade Católica Dom Bosco, Programa de Pós-Graduação em Psicologia, Av. Tamandaré, 6000, Jardim Seminário, Campo Grande (MS), CEP: 79117-900 I Email: rlmiranda@ucdb.brl doi: 10.18761/PAC.2020.v11.n1.01

Resumo: Estudos em História da Análise do Comportamento, no Brasil, têm sido debatidos com foco, especialmente, nos anos iniciais de conformação da sua comunidade científica. Este trabalho buscou apresentar a Associação de Modificação do Comportamento (AMC). Fundada em 1974 e extinta em 1984, com sede e foro no estado de São Paulo, a AMC teve como objetivo a promoção da modificação do comportamento como campo científico e profissional. Reuniu associados, promoveu eventos e publicou revistas especializadas. Nesse sentido, parte desses documentos foi fonte de dados do estudo em questão, cuja finalidade foi tentar conhecer o perfil dessas pessoas, ou seja: quem eram, o que liam e o sobre o que falavam. Os dados apontaram para a predominância do gênero feminino, da escrita singular, de filiações do estado de São Paulo, de interesse em temas educacionais e de destaque para a área aplicada. Houve, ainda, colaborações de atores internacionais (Canadá, Estados Unidos e México). Ainda, limitações metodológicas precisam ser levadas em consideração, sem, no entanto, preterir o fato deque os resultados desta pesquisa proporcionam conjecturas para uma compreensão mais sofisticada da Análise do Comportamento, no Brasil, bem como auxiliam na preservação de sua memória.

Palavras-chave: História da Psicologia; Análise do Comportamento; Bibliometria; Sociedades Científicas.

1 Doutoranda pela Universidade Católica Dom Bosco e bolsista (Processo n ${ }^{\circ}$ 88887.485254/2020-00 do Programa PROSUC/CAPES) 
Abstract: Studies in History of Behavior Analysis, in Brazil, have been debated with focus especially on the initial years of its scientific community conformation. This research aimed to present the Associação de Modificação do Comportamento (AMC). Founded in 1974 and extinguished in 1984, with headquarters and operations in the state of São Paulo, AMC intended to promote behavior modification as a scientific and professional field. It gathered members, promoted events and published specialized journals. In this sense, part of those documents was the data source of this study, whose purpose was trying to know the profile of those people, that is: who they were, what they read and what they talked about. The data pointed to predominance of the feminine gender, singular writing, affiliations from the state of São Paulo, interest in educational topics and emphasis on applied area. There were also collaborations from international actors (Canada, United States and Mexico). Still, methodological limitations need to be taken into account, without, however, neglecting the fact that the results of this research provide conjectures for a more sophisticated understanding of Behavior Analysis in Brazil, as well as assisting in the preservation of its memory.

Keywords: History of Psychology; Behavior Analysis; Bibliometrics; Scientific Society.

Resumen: Estudios en Historia del Análisis de la Conducta, en Brasil, han sido debatidos con enfoque, especialmente, en los años iniciales de conformación de su comunidad científica. Este trabajo buscó presentar la Associação de Modificação do Comportamento (AMC). Fundada en 1974 y extinta en 1984, con sede y foro en el estado de São Paulo, AMC tuvo como objetivo la promoción de modificación de la conducta como campo científico y profesional. Reunió asociados, promovió eventos y publicó revistas especializadas. Parte de esos documentos ha sido fuente de datos de este estudio, cuya finalidad fue intentar conocer el perfil de esas personas, o sea: quiénes eran, qué leían y de qué hablaban. Los datos apuntaron a la predominancia del género femenino, de la escritura singular, de filiaciones del estado de São Paulo, de interés en temáticas educacionales y de destaque para la aplicación. También, hubo colaboraciones de actores internacionales (Canadá, Estados Unidos y México). Sin embargo, las limitaciones metodológicas necesitan ser tenidas en cuenta, sin, mientras tanto, preterir el hecho de que los resultados de esta investigación proporcionan conjeturas para una comprensión más sofisticada del Análisis de la Conducta, en Brasil, así como ayudan en la preservación de su memoria.

Palabras clave: Historia de la Psicología; Análisis de la Conducta; Bibliometria; Sociedad científica. 
De acordo com alguns autores, o desenvolvimento da Análise do Comportamento tem convidado à reflexão historiográfica e, com isso, o interesse sobre aspectos históricos de tal disciplina tem aumentado (Cruz, 2006; Morris, Todd, Midgley, Schneider \& Johnson, 1990). Consequentemente, os escritos sobre a história da Análise do Comportamento têm se avolumado, produzindo uma literatura especializada sobre o campo. No Brasil, a história da área é extensamente discutida, principalmente no que se refere aos seus anos iniciais de institucionalização. Os temas estudados são variados, como os primeiros cursos de Análise do Comportamento (Cândido, 2017a; Cirino, Miranda \& Cruz, 2012; Cirino, Miranda \& Souza Júnior, 2012; Guedes et al., 2006; Matos, 1998; Miranda, 2010; Todorov, 2006; Todorov \& Hanna, 2010), a aplicação na educação (Akera, 2017; Cândido, 2017b; Nale, 1998; Souza Júnior, Miranda \& Cirino, 2018), a contribuição de personagens (Cândido \& Massimi, 2016; Peron, Nogueira, Cândido, \& Massimi, 2015; Tomanari, 2005) e a circulação da Análise do Comportamento em diferentes estados (Souza, 2011; Val, 2013).

Tais trabalhos apontam para o que a História e a Sociologia das Ciências vêm discutindo há algum tempo: o funcionamento da ciência, a partir da conformação de comunidades científicas (e.g., Fleck, 1935/2010; Knorr-Cetina, 1999). Essas comunidades produzem mecanismos de institucionalização de sua disciplina, pela produção de condições de ensino, criação de manuais didáticos, circulação de periódicos especializados, promoção de associações específicas, produção de eventos, e outros. A partir desses mecanismos, elas delimitam, ao longo de sua história, regras que conformam e configuram sua organização e práticas investigativas (Rosa, Huertas, \& Blanco, 1996). Assim, o exame de aspectos da institucionalização da disciplina, explorando relações entre eles e o seu contexto de produção, configura-se numa possibilidade de fazer história das ciências.

Apesar do grande esforço em produzir conhecimento sobre a história da Análise do Comportamento, no Brasil, a contribuição das associações científicas ainda é pouco explorada. A única exceção é a análise do papel da
Associação Brasileira de Psicologia e Medicina Comportamental (ABPMC), cujas contribuições para o ensino, a pesquisa, a divulgação e a aplicação já foram narradas (Guedes, Guimarães \& Queiroz, 2007). Nesse cenário, pouco se conhece sobre as contribuições daquelas que antecederam a ABPMC, como é o caso da Associação de Modificação do Comportamento (AMC), fundada em 1974 e sucedida, em 1985, pela Associação Brasileira de Análise do Comportamento (ABAC). Diante disso, o objetivo deste trabalho foi descrever e analisar características da comunidade de analistas do comportamento vinculados à AMC. Particularmente, focam-se nos textos publicados por seu periódico, Modificação do Comportamento: Pesquisa e Aplicação. Metodologicamente, a investigação utilizou documentos primários, produzidos pela referida associação e acessados junto aos arquivos do Laboratório de Estudos Históricos em Análise do Comportamento (LeHac-PUC/SP). Nesse sentido, foram analisados: (a) cartas informativas Modificação do Comportamento, não vinculadas à AMC, embora tenham divulgado a sua fundação; (b) o Estatuto da AMC; (c) seus boletins informativos e (d) os dois números da Revista Modificação do Comportamento: Pesquisa e Aplicação, publicadas pela AMC.

Para atingir o objetivo proposto, o texto está dividido em três seções: (a) condições para a criação da AMC, (b) descrição de aspectos da Associação e (c) caracterização do periódico, salientando os aspectos da comunidade científica ali presentes (e.g., quem eram, o que liam, sobre o que falavam, etc.). Ao final, estima-se apresentar papéis desempenhados pela Associação, no processo de institucionalização da área, no Brasil. Esses resultados coadunam com a literatura em História das Ciências (e.g., Rosa et al., 1996), bem como com aquilo que Witter (2007) indica como importante no estudo historiográfico de instituições científicas: "A importância das Sociedades Associações/Científicas decorre delas gerarem e preservarem a História da Ciência e das Profissões relacionadas, de criarem estímulos e condições de desenvolvimento, quer da ciência, quer da profissão" (p. 3). 


\section{Condições para a criação da AMC}

Os anos de 1960 marcam o início da institucionalização da Análise do Comportamento, no Brasil, com a ocasião da vinda de Fred Simmons Keller à Universidade de São Paulo (USP) (Cândido, 2017b; Matos, 1998; Sabadini, 2013). Esse período registra, também, a regulamentação da profissão e formação em Psicologia, com a sanção da Lei Federal N. ${ }^{\circ}$ 4.119, em 1962. Em 1961, Keller e sua esposa, Frances, foram recepcionados por um grupo no qual estavam, entre outros, Carolina Martuscelli Bori e Rodolpho Azzi, designadospara exercer a função de assistentes de Keller. Em 1962, Gil Sherman substituiu Fred Keller em suas atividades, junto à USP (Akera, 2017; Todorov, 2006). A atuação conjunta desses quatro professores foi fundamental para a formação de um primeiro grupo de analistas do comportamento, em diferentes regiões do país. Cite-se, como exemplo, o fato de que eles ministraram aulas em Rio Claro (São Paulo, SP), entre 1962 e 1963, e em Brasília a partir de 1964 (Cândido, 2017a; Todorov \& Hanna, 2010). Todavia, com a ascenção do governo ditatorial civil-militar, houve intervenção direta na Universidade de Brasília (UnB) e os professores acusados de serem "comunistas" foram cassados. Nesse cenário, vários analistas do comportamento se afastaram dessa universidade e se espalharam por outros estados brasileiros e, alguns, por outros países. Esse período ficou conhecido como diáspora da Análise do Comportamento, no Brasil, quando a área se espalhou em diferentes instituições do país (Guedes et al., 2008; Todorov, 2006; Todorov \& Hanna, 2010).

A institucionalização da Análise do Comportamento compõe o cenário vivido pela Psicologia, no Brasil, à época. A década de 1960 marcou o crescimento na instalação de cursos de graduação e, consequentemente, houve um crescimento do número de registros profissionais, em Psicologia, dentre outros (Pereira \& Pereira Neto, 2003; Rosas, Rosas, \& Xavier, 1988). Foi em 1962 que ocorreu a regulamentação da formação e profissão de psicólogo (Lei Federal No. 4.119, 1962), com participações de analistas do comportamento (e.g., Carolina Bori). A partir dessas mudanças, instalou-se, na década de 1970, um cenário de preocupação com as ativi- dades éticas da Psicologia - principalmente aquelas vinculadas a suas aplicações - e com sua organização em sociedades científicas. Novamente, brasileiros vinculados à Análise do Comportamento participaram desse processo. Estudantes de Psicologia da USP, campus Ribeirão Preto, aliaram-se a alguns professores (e.g., João Cláudio Todorov, Frederico Graeff, Reinier Rozestraten) e, juntos, criaram uma sociedade científica e profissional que se concretizou como Sociedade de Psicologia de Ribeirão Preto (SPRP), hoje Sociedade Brasileira de Psicologia (Gorayeb, 1991; Rubiano, 2005). Esse grupo, ligado à criação da SPRP, em Ribeirão Preto, fez circular a Carta Informativa Modificação do Comportamento, que tinha o objetivo de "propiciar contato entre os profissionais que trabalham com Modificação do Comportamento ou que são responsáveis pela formação de psicólogos que trabalharão nesta área" (Modificação de Comportamento, 1974a, n. 2). Foi nesse contexto de preocupação com as questões aplicadas que surgiram, também, as primeiras aproximações entre a Análise do Comportamento com as questões de saúde, em solo brasileiro.

No Brasil, uma primeira aproximação se deu durante o curso de Modificação do Comportamento que ocorreu na Universidade Católica de Campinas, em 1969, sob a responsabilidade de Luiz Otávio Seixas Queiroz. O conteúdo incluía as dimensões da Análise do Comportamento Aplicada (em inglês, Applied Behavior Analysis - ABA), associando procedimentos clínicos e pesquisa experimental (Queiroz \& Guilhardi, 1980). Segundo a literatura, essa experiência foi decisiva no estabelecimento de uma formação mais ampla, em Modificação do Comportamento, que aconteceria, anos mais tarde, na Pontifícia Universidade Católica de São Paulo (PUC-SP) (Queiroz, Guilhardi, Martin, \& Guedes, 1976). Além de Luiz Otávio Seixas Queiroz, o curso da PUC-SP contava com Hélio Guilhardi - que havia sido aluno, em Campinas - e Garry Martin, professor da Universidade de Manitoba (Canadá), que forneceu importantes contribuições para a construção da Modificação do Comportamento como uma área de aplicação e construção de conhecimento. Além desses dois cursos, realizou-se, na cidade de São Paulo, um curso sobre Modificação Cognitiva do Comportamento, ministrado por Michael Mahoney (Leonardi, 2015). Foi a partir desses cur- 
sos que se formou o grupo que esteve à frente da AMC, como registrado na Carta Informativa n. 5 (Modificação de Comportamento, 1974b): "No dia 25 de novembro p.p. realizou-se uma reunião na PUC-SP, sob a coordenação do Professor Garry L. Martin, com o objetivo de criar uma Associação de Modificadores de Comportamento, no Brasil, a exemplo da experiência realizada em Manitoba, Canadá” (p. 3).

\section{Sobre a Associação de Modificação do Comportamento}

Em novembro de 1974, foi fundada a AMC, entidade civil sem fins lucrativos, com sede e foro no município de São Paulo, estado de São Paulo. Em sua fundação, ela foi organizada para funcionar por meio de um Conselho de Coordenadores definido por votação, em assembleia. Hierarquicamente abaixo desse Conselho, estavam os seguintes comitês: de Publicações; de Congresso; de Relações Públicas; de Assuntos Legais e Éticos; de Secretaria e de Eleições (Associação de Modificação do Comportamento, 1975a). No que tange à associação, poderiam ser sócios aqueles que estivessem em uma das três categorias: (i) assinantes da ata de fundação, (ii) aqueles que foram indicados por um dos associados ou (iii) aqueles que demonstrassem interesse, devendo o sócio contribuir com a associação mediante o pagamento de anuidades. Os associados poderiam votar e se voluntariar para trabalhar nos referidos comitês, além de receberem publicações periódicas da associação. Em setembro de 1975, a AMC contava com, aproximadamente, 247 associados (Associação de Modificação do Comportamento, 1975b). Não foi possível identificar a unidade federativa dos associados, mas notou-se que, daquele total, a maioria era composta por mulheres $(n=191)^{1}$. Como se verá ao longo deste texto, essa é uma característica marcante da comunidade analítico-comportamental, vinculada à AMC. Apesar de não se encontrar fontes primárias que sinalizassem quem estava envolvido com a criação da AMC, nota-se, em um de seus Boletins,

1 Foram elencados 48 nomes masculinos, 191 femininos e nove cujos gêneros não foram identificados por serem em línguas orientais ou abreviados. um conjunto de nomes referentes aos envolvidos em sua gestão. Quase a totalidade dos nomes que compunham os comitês eram femininos - apenas três, de 28 nomes, não o eram.

Em relação a seu estatuto, é possível observar os objetivos: “(a) o desenvolvimento da modificação do comportamento como ciência; (b) o desenvolvimento da modificação do comportamento como profissão; (c) o desenvolvimento da modificação do comportamento como um meio de promover o bem-estar humano" (Associação de Modificação do Comportamento, 1975a, p. 1). Ainda de acordo com o referido documento, observa-se que a associação se organizava de forma a promover a modificação do comportamento, tanto como campo científico quanto profissional, com o objetivo de promoção do bem-estar das pessoas. Isso era proposto por meio do desenvolvimento de pesquisas, da divulgação de materiais e do cuidado com "a qualidade do serviço de modificação do comportamento" (Associação de Modificação do Comportamento, 1975a, p. 1). Nesse sentido, para atingir tais metas, a AMC já previa, em seu estatuto, entre outras ações, a criação de um periódico específico.

Em maio de 1975, no Boletim Informativo da AMC, circulou a notícia de que o Comitê de Publicações se envolvera com a "criação, publicação e manutenção de uma revista especializada em artigos sobre modificação do comportamento" (Associação de Modificação do Comportamento, 1975c, p. 5). Pouco mais de um ano depois, em outubro de 1976, foi publicado o periódico científico intitulado Modificação de Comportamento: Pesquisa e Aplicação. Sua vigência foi curta, com apenas dois números publicados, sendo um ainda em 1976 e o segundo, em abril de 1977.

\section{Sobre Modificação de Comportamento: Pesquisa e Aplicação}

A Modificação de Comportamento: Pesquisa e Aplicação foi uma revista científica editada pela AMC e publicada pela Editora de Humanismo, Ciência e Tecnologia (HUCITEC). À época, a editora foi responsável pela publicação de outros ma- 
teriais vinculados à Análise do Comportamento, como, por exemplo, o livro Princípios do Comportamento (Ferster, Culbertson, \& Perrott Boren, 1978) e a revista Psicologia. Esse periódico, inclusive, também esteve relacionado à institucionalização da Análise do Comportamento, no país, sendo veiculado como uma revista de potencial interesse aos associados da AMC (Associação de Modificação do Comportamento, 1975d). O primeiro número da Modificação de Comportamento: Pesquisa e Aplicação teve como editor Sérgio Luna; o segundo número contou com Adalgisa Pereira da Silva e Flávio George Aderaldo, na editoração. Luna era professor, na PUC-SP, enquanto Silva e Aderaldo estavam vinculados à própria HUCITEC.

A Tabela 1 apresenta a compilação dos 11 textos que foram publicados, mais o Manual para Preparação de Originais, que pretendia servir de complemento ao Manual da American Psychological Association, em uma tentativa de "tornar explícitas e detalhadas as informações desse manual" (Comitê de Publicações, 1977, p. 119). Essa explanação, em um documento à parte, parecia guardar relação com o fato de que os responsáveis pelo periódico esperavam receber contribuições de estudantes e pesquisadores iniciantes (Comitê de Publicações, 1977). Todas as publicações foram escritas na língua portuguesa do Brasil, sendo que uma delas, original da Universidade de Manitoba, foi traduzida por Sonia Maria Scala Padalino. A respeito da participação simultânea, na autoria de qualquer uma das publicações e na edição do periódico e/ ou no quadro representativo da AMC, houve apenas uma ocorrência, na segunda edição: Hélio José Guilhardi foi o primeiro autor do sexto texto publicado e esteve envolvido na edição daquele número.

Trabalhos publicados no periódico Modificação de Comportamento: Pesquisa e Aplicação (1976-1977)

\begin{tabular}{|c|c|c|}
\hline \multirow[b]{6}{*}{$\begin{array}{l}\stackrel{0}{\circ} \\
\stackrel{0}{\circ}\end{array}$} & Título & Autores \\
\hline & $\begin{array}{l}\text { Efeitos de um programa de matemática nos comportamentos } \\
\text { da professora e dos alunos de uma classe de segundo ano } \\
\text { do } 1 .^{\circ} \text { grau }\end{array}$ & $\begin{array}{l}\text { Sérgio Antônio da Silva Leite; Potiguara Tadeu } \\
\text { Bezerra Bastos; Norma Suely Collado Bastos }\end{array}$ \\
\hline & Efeito do local de estudo no comportamento de estudar & Maria Olímpia Jabur \\
\hline & A pseudo-artificialidade de sistemas de vales & Célia Maria C. Gonçalves \\
\hline & A Análise do comportamento aplicada à Escola & Nilce Pinheiro Mejias \\
\hline & $\begin{array}{l}\text { Controle de uma cadeia de respostas de colocação de obje- } \\
\text { tos em série, em crianças de cinco anos de idade }\end{array}$ & $\begin{array}{l}\text { Mariuza Pelloso Lima; Ivan Antônio de Oliveira; José } \\
\text { Antônio Küller }\end{array}$ \\
\hline \multirow{7}{*}{ ๙ิ } & $\begin{array}{l}\text { Aumento de freqüência de respostas acadêmicas para alterar } \\
\text { a lentidão e eliminar comportamentos inadequados em um } \\
\text { aluno de primeiro grau }\end{array}$ & $\begin{array}{l}\text { Hélio José Guilhardi; Maria Estela SigristBetini; } \\
\text { Maria Cecília dos Santos Camargo }\end{array}$ \\
\hline & $\begin{array}{l}\text { Redução da enurese noturna infantil: Considerações sobre } \\
\text { os efeitos do procedimento de micções noturnas planejadas } \\
\text { em intervalos de tempo gradualmente aumentados }\end{array}$ & Vera Rebouças Pereira de Almeida \\
\hline & $\begin{array}{l}\text { Efeitos de um programa remediativo de alfabetização nos } \\
\text { comportamentos de crianças da } 1 .^{a} \text { série e da professora }\end{array}$ & $\begin{array}{l}\text { Sérgio Antônio da Silva Leite; Marilena Kerches } \\
\text { de O. Silva Leite; Vera Lúcia Ramires; Zulma } \\
\text { Lane Inácio Marcondes; Maria Aparecida Araújo } \\
\text { Guimarães }\end{array}$ \\
\hline & $\begin{array}{l}\text { Modificação de comportamento de inibição (ou de isolamen- } \\
\text { to) de criança em idade escolar - Estudo de um caso }\end{array}$ & Dagmar M. L. Zibas \\
\hline & $\begin{array}{l}\text { O uso do reforçamento diferencial no treinamento de profes- } \\
\text { sores: Um programa para instalar o comportamento de apre- } \\
\text { sentação de conseqüências imediatas e explícitas ao aluno }\end{array}$ & Elias Moacir da Costa \\
\hline & Uma revisão do sistema de fichas na pesquisa aplicada & Eugene Anthony Kaprowy \\
\hline & Manual para a preparação de originais & Comitê de Publicações da AMC \\
\hline
\end{tabular}




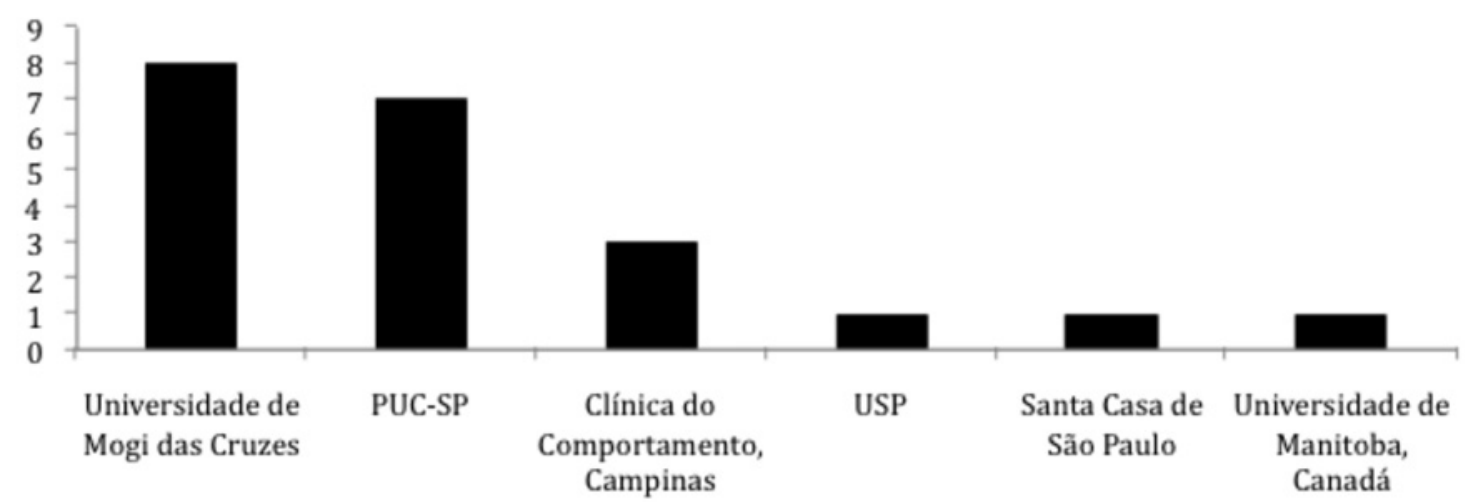

Figura 1. Instituições de filiação de quem publicou no periódico Modificação de Comportamento: Pesquisa e Aplicação, conforme número de autores.

\section{Quem eram?}

Como se vê na Tabela 1, os 11 artigos publicados nos dois números do periódico foram assinados, ao total, por 21 autores. Quanto ao gênero das autorias, de um total de 21 autores e coautores, a maioria referia-se a nomes atribuídos a mulheres $(n=13)$. A forte presença feminina refletia o que já vinha sendo observado na constituição da AMC e ao encontro daquilo que Keller (1988) destacou, ao apresentar nomes que poderiam ser encontrados no quadro da AMC e nas publicações de seus periódicos (e.g., Carolina Bori e Maria Amélia Matos). Por fim, esse resultado coaduna com o perfil tradicionalmente feminino na Psicologia, no Brasil. A literatura nos indica que, desde a década de 1950, havia preponderância feminina em publicações relacionadas à Psicologia (Mota, Castro, \& Miranda, 2016; Mota \& Miranda, 2017). Na década de 1980, no campo profissional, havia um total de $87 \%$ de psicólogas registradas no Conselho Federal de Psicologia (Conselho Federal de Psicologia, 2013).

No que tange à colaboração na escrita, sete textos foram elaborados por apenas uma pessoa e quatro foram produzidos por duas pessoas, ou mais. Esse padrão de escrita singular parece ser historicamente prevalente em publicações relacionadas à Psicologia, no país (Mota et al., 2016; Mota \& Miranda, 2017). Considerando as filiações institucionais apresentadas nos manuscritos, nota-se que as escritas coletivas não apresentaram filiação em mais de uma instituição (i.e., cada um desses textos foi produzido em uma única instituição). Os autores estavam em seis instituições (Figura 1) e se observa, inclusive, que a maior parte deles se concentra em apenas duas: Universidade de Mogi das Cruzes e PUC-SP ${ }^{2}$. Um ponto em comum entre as duas era o fato de que vinham realizando trabalhos de modificação do comportamento, desde 1973 (Cury, 1996; Modificação de Comportamento, 1974a). Na primeira, destacou-se o trabalho coordenado por Sérgio Leite com grupos escolares e, na segunda, a modificação do comportamento foi trabalhada com Rachel Kerbauy. Além disso, na PUC-SP, também estava ocorrendo um curso de Modificação do Comportamento junto à graduação, em Psicologia, sob a responsabilidade de Garry Martin, Luiz Otávio Queiroz e Hélio Guilhardi. Dessa forma, se o trabalho vinha sendo realizado há alguns anos, já poderia haver resultados a serem publicados.

A Figura 1 compila as instituições de filiação dos autores e coautores, permitindo ver que quase todas eram provenientes do estado de São Paulo (SP) $(n=10)$, sendo apenas uma do Canadá. Apesar de não haver indicativos de que a AMC era uma associação estadual, considerando seu estatuto, pode-se afirmar um foco em São Paulo (Botomé, 2006). Isso se deve ao fato de que a revista era editorada, no estado, pela HUCITEC ou, simplesmente,

2 Embora a referência seja à PUC-SP, quanto à atuação de Kerbauy, vale lembrar que o início da década de 1970 foi um momento em que a Faculdade de Filosofia, Ciências e Letras Sedes Sapientae (onde ela trabalhava) era incorporada à PUC-SP. 
pela ausência de pessoas trabalhando, na área, em outras regiões do país. Essa segunda hipótese necessita de maiores investigações para se concretizar como uma afirmação.

Ao observar, novamente, o estatuto da AMC, constata-se que uma das atribuições do Conselho de Publicações era "convidar pessoas a mandarem artigos como possível publicações em revistas da Associação" (sic) (Associação de Modificação do Comportamento, 1975a, p. 4). Dessa forma, se havia "convite" aos autores, mesmo se houvesse revisão por pares, havia maior probabilidade de que tal acontecesse no estado de SP, já que era um círculo de relação fisicamente mais próximo. Em relação à presença de uma instituição canadense, a Universidade de Manitoba, nota-se a influência de Garry Martin junto ao grupo que estava à frente da AMC.

\section{O que e quem liam?}

Os 11 textos publicados no Modificação de Comportamento: Pesquisa e Aplicação citaram um total 143 referências, sem excluir repetições de materiais. Desse total, 102 foram consultas a periódicos; os livros apareceram em 36 ocorrências e outros tipos de material totalizaram 5. No que se refere aos periódicos, chama a atenção a frequência de ocorrência do Journal of Applied Behavior Analysis [JABA] $(n=72)$. O JABA foi um periódico criado por aqueles envolvidos com a comunidade analítico-comportamental estadunidense, interessada em aspectos aplicados do campo, uma vez que o grupo apontado tinha dificuldades em circular suas produções nos Estados Unidos da América EUA (Wolf, 1993). Ademais, Wolf (1993) sinaliza que outro periódico, no qual a comunidade poderia publicar seus textos, era o Behavior Research and Therapy (BRT), um periódico britânico vinculado a Hans Eysenck, um dos precursores das terapias comportamentais. Assim, não parece ser por acaso que a BRT foi a segunda revista mais frequente $(n=5)$ em relação a publicações nessa área. As próximas quatro revistas mais frequentes eram vinculadas, fortemente, aos campos da Psicologia do Desenvolvimento e da Psicologia da Educação, como, por exemplo, Child Development $(n=3)$ e Psychology in the School $(n=3)$.
Do total de materiais citados, a maior parte estava em outros idiomas $(n=122)$ - especialmente a língua inglesa $(n=119)$ - e apenas algumas em língua espanhola do México $(n=3)$. Pode-se entender a prevalência de citações em inglês, a partir das conexões entre Brasil e EUA, à época. Ao observar os nomes dos autores citados, dentre aquelas 143 referências, chama a atenção o fato de que houve apenas uma menção a obras de Burrhus Frederic Skinner (1904-1990) - Contingencies of reinforcement: a theoretical analysis - e duas às de Keller: Good-bye teacher e Um curso moderno de Psicologia. Entre os autores mais citados, Sérgio Antônio da Silva Leite aparece entre os dez mais consultados, mas as evidências sugerem que isso ocorreu devido às autocitações (Figura 2).

Na Figura 2, há destaque para estudiosos das aplicações da Análise do Comportamento como, por exemplo, Montrose Wolf e Vance Hall ${ }^{3}$. O primeiro foi um influente vetor no estabelecimento da ABA como um "braço" da Análise do Comportamento, em um momento de controvérsias do campo (Baer, Wolf, \& Risley, 1968; Wolf, 1993). O segundo, no campo das aplicações clínicas, chegou a ter obras traduzidas de modificação e manipulação do comportamento, no Brasil (Hall, 1973). Chama a atenção, também, a presença de Charles Ferster que, à época, vinha discutindo aspectos de psicoterapia relacionados à Análise do Comportamento (Ferster, 1972). É interessante observar que a influência de tais autores se deu, também, por sua presença, no Brasil. Em julho de 1974, Ferster e Donald Baer estiveram no Brasil, em Belo Horizonte e em São Paulo (Modificação de Comportamento, 1974b). Baer, em uma entrevista concedida à AMC, em 1975, indicou uma percepção positiva sobre os conhecimentos dos modificadores do comportamento, no país. Ele salientou, inclusive, que as aplicações brasileiras estavam pari passu àquelas que ele observava alhures. Dessa forma, percebem-se influências do campo aplicado da Análise do Comportamento na AMC. Isso ocorreu

3 Optou-se por fazer uma distinção entre a aplicação da Análise do Comportamento e a ABA. O primeiro se referiria à inserção da Análise do Comportamento em contexto aplicado (e.g., escolas, psicoterapia, etc.) e o segundo seria a utilização da ABA, isto é, aplicações que respeitassem as oito características definidas por Baer, Wolf e Risley (1968). 


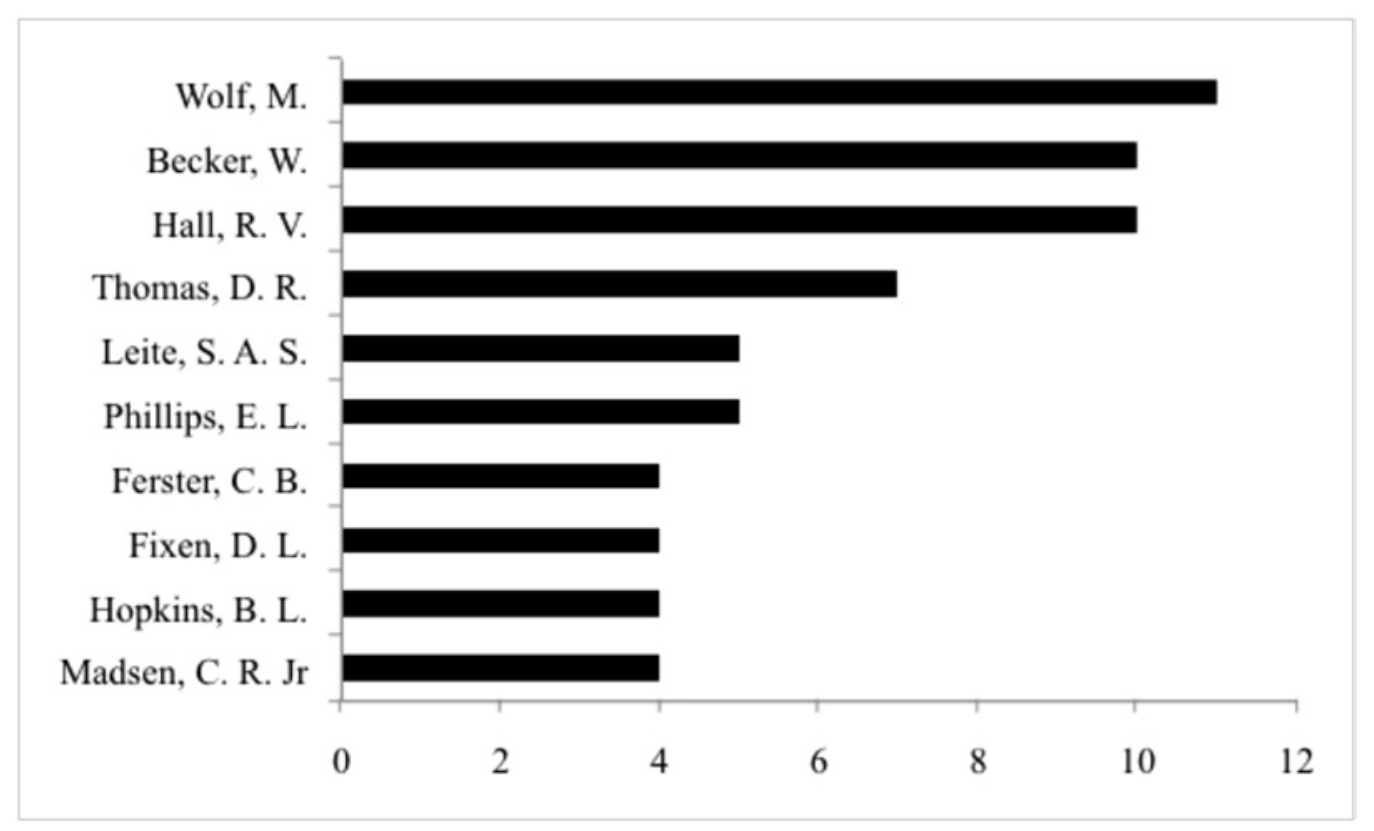

Figura 2. Os dez autores mais consultados por quem publicou no periódico Modificação de Comportamento: Pesquisa e Aplicação, conforme quantidade de citações nas referências.

tanto pela leitura e citação de tal material quanto pela visita de alguns daqueles autores ao Brasil.

Quanto às dez obras mais consultadas, depreende-se que todas elas têm relação com o campo educacional, desde o estabelecimento de contingências para a educação infantil até o trabalho com comportamentos disruptivos, em sala de aula. Como será possível constatar mais à frente, aqueles que publicavam na Modificação de Comportamento: Pesquisa e Aplicação estavam fortemente interessados nas aplicações da Análise do Comportamento na educação. Isso também será observado nos interesses mais gerais daqueles vinculados à própria AMC. O livro mais citado é Rules, praise, and ignoring: elements of elementary classroom control, de Madsen, Becker e Thomas (1968). Todos esses autores estão, também, entre os dez mais citados. Isso pode sugerir que, além dos autores serem influentes, no geral, eles possuíam obras de referência específica para aqueles que publicavam no periódico. Cite-se, a título de exemplo, que Don Thomas é o terceiro autor mais citado $(n=7)$ e, desse total, duas de suas obras estão entre as mais mencionadas. Isso pode sugerir não apenas que suas obras se tornaram referências, já que estão entre as mais citadas, mas que ele próprio era uma referência, uma vez que ocorria ser consultado em mais de um trabalho. Outro exemplo é Montrose Wolf, que foi o autor mais citado, porém não teve uma obra dentre as mais recorrentes. Isso pode sugerir, igualmente, que variados trabalhos de sua autoria consistiam em referências para os brasileiros, já que não houve concentração em apenas uma obra.

Esses resultados demonstram uma ampliação do campo em relação ao que se fazia até 1969, quando a maior parte da atuação na área esteve focada em estudos de laboratório de AEC e educação. Apesar de haver um grande número de artigos sobre educação, aqueles publicados nessa revista não mais focavam o método de ensino em si, mas incluíam problemas individuais que interferiam na aprendizagem. Ao analisar os nomes dos autores mais citados, pode-se afirmar, seguramente, que havia uma preocupação com a aplicação do conhecimento. A partir das revistas científicas de artigos citados, há de se reconhecer uma forte influência dos trabalhos que se publicavam no JABA, seguida de um interesse por terapia. Vale considerar, ainda, a presença de Ribes-Iñesta, do México, que representou uma ampliação da Análise do Comportamento, ao redor do continente americano. 


\section{Sobre o que falavam?}

A apreciação dos temas tratados em Modificação do Comportamento: Pesquisa e Aplicação aponta a Educação como uma grande área de interesse, dando continuidade ao que se vinha diligenciando em Análise do Comportamento, no país, naquela época (Akera, 2017; Cirino, Miranda, \& Cruz, 2012; Cirino, Miranda, \& Souza Júnior, 2012; Souza Júnior et al., 2018). Nessa seara, notam-se desde trabalhos de intervenção a reflexões teórico-conceituais. No campo da intervenção, vê-se um interesse por comportamentos disruptivos, ou inadequados, em ambiente escolar (e.g., Guilhardi, Betini, \& Camargo, 1977; Jabur, 1976; Leite, Bastos, \& Bastos, 1976; Zibas, 1977). Esses trabalhos procuravam, via de regra, diminuir a frequência de comportamentos inadequados por meio do foco no aumento do desempenho acadêmico de crianças. Esse tipo de intervenção, com estabelecimento de concorrentes ao comportamento-queixa, parecia ir de encontro ao que se assemelhava ser mais comum à época: "Maioria dos estudos experimentais divulgados tem considerado o problema da manipulação de comportamentos inadaptados em sala de aula através do uso de reforçamento social ... enquanto que o desempenho acadêmico tem recebido pouca atenção" (Leite et al., 1976, p. 2).

$\mathrm{Na}$ mesma direção, outro artigo delineou seus objetivos: "Trabalhar diretamente com o desempenho acadêmico do aluno e, paralelamente, observar o que ocorre com os comportamentos inadequados" (Guilhardi et al., 1977, p. 3). Complementando os trabalhos de intervenção, havia outros que refletiam sobre o lugar da Análise do Comportamento aplicada à escola:

Nós não temos, como nas escolas americanas, uma tradição de formação do psicólogo escolar nos moldes do psicólogo clínico. Aliás, não nos parece que tenhamos ainda uma tradição de formação do psicólogo escolar. E a própria presença do psicólogo, em nossas escolas, ainda é uma exceção e quase um privilégio de instituições particulares de nível sócio-econômico elevado. (Mejias, 1976, p. 48)
Dessa forma, parece que os trabalhos na grande área da educação procuravam não apenas estabelecer aplicações da Análise do Comportamento, para comportamentos relevantes, como, também, refletir sobre a própria Análise do Comportamento e, eventualmente, sobre a própria Psicologia, no contexto escolar.

Quando se evidenciou o tipo de pesquisa pelos seus procedimentos, cuja prevalência foi de delineamentos experimentais, atentou-se que tais investigações também ocorriam em contexto educacional (e.g., Costa, 1977; Jabur, 1976; Leite et al., 1976; Leite, Leite, Ramires, Marcondes, \& Guimarães, 1977). Os participantes eram tanto as crianças, alunas das escolas, quanto os seus professores. Todavia, a maioria dos estudos procuravam observar os efeitos de arranjos de diferentes contingências nas respostas das crianças, como, por exemplo, na mudança do comportamento de estudar (Jabur, 1976) ou na ampliação de repertórios de estudo concorrentes ao responder inadequado (Guilhardi et al., 1977). Assim, o repertório dos professores ficava em segundo plano, com apenas um estudo, cujo objetivo foi observar os efeitos de um treino de alfabetização sobre o repertório da professora (Leite et al., 1977).

Ao focalizar os tipos das pesquisas quanto à sua natureza, vê-se que a aplicação da Análise do Comportamento, embora recorrente em ambiente escolar, também aparecia a partir de estudos de caso (e.g., Almeida, 1977; Gonçalves, 1976; Zibas, 1977). O texto de Gonçalves (1976), por exemplo, discutia características da economia de fichas (em inglês, token economy) como possibilidade de manejo comportamental em contexto aplicado. A autora, mais especificamente, refletia sobre o fato de que tal procedimento não é, necessariamente, artificial. Nas palavras da autora:

A diferença entre cada uma das possíveis áreas de aplicação de sistema de vales (escola, hospital psiquiátrico, prisões, instituições para excepcionais) encontra-se nas classes de comportamentos escolhidos e/ou nas categorias de consequências e $S^{\mathrm{D}}$ 's e não manter relação desses componentes com as fichas ou pontos entre eles. Ou seja, o arranjo de contingências deveria ter objetivos comuns. (Gonçalves, 1976, p. 38) 
Outro exemplo é o texto de Almeida (1977), cujo trabalho procurava intervir em casos de enurese noturna. Esse estudo envolveu orientação às mães das crianças, a fim de que cumprissem o papel inicial de observadoras e, então, colocassem em prática a técnica de chamamentos noturnos. Devido a questões éticas e técnicas (e.g., tempo para execução), não foi possível haver um controle experimental e, por esse motivo, a pesquisa foi considerada um estudo de caso.

Assim, notou-se que o conjunto de autores que publicava no Modificação de Comportamento: Pesquisa e Aplicação parecia se preocupar, predominantemente, com temas educacionais, com destaque para a diminuição de frequência de comportamentos inadequados. Para tanto, o foco esteve no desempenho acadêmico. Todos os trabalhos experimentais tinham, como meta, a mudança de comportamento de menores de idade, mesmo na única pesquisa em que os sujeitos eram os professores. $\mathrm{O}$ sistema de fichas foi defendido como uma técnica eficiente da área, apesar de apresentar alguns problemas e de serem necessárias pesquisas adicionais. Os trabalhos foram, majoritariamente, do tipo experimental e todos apresentaram natureza aplicada.

\section{Considerações Finais}

Os anos iniciais da Análise do Comportamento, no Brasil, foram marcados pela criação de condições de pesquisa experimental e formação de pesquisadores. A criação da AMC contribuiu, principalmente, para a institucionalização da Análise do Comportamento como uma área de aplicação. Apesar da grande preocupação com problemas educacionais, o que se observa é o debate de temas que vão além daqueles ligados aos métodos de ensino, como no Personalized System of Instruction, ensino programado. Os artigos publicados em Modificação do Comportamento: Pesquisa e Aplicação abordam problemas de comportamento, em sala de aula, buscando auxiliar no desenvolvimento de comportamentos mais adaptados. Particularmente, as informações coletadas nos dois números de Modificação de Comportamento: Pesquisa e Aplicação sugerem que a comunidade de analistas do comportamento estava ocupada com questões educacionais, es- pecialmente com a diminuição de frequências de comportamentos inadequados. As pesquisas com sistemas de fichas corroboravam inferências no sentido de auxiliar crianças e adolescentes, pela diminuição de comportamentos-problema.

Havia uma forte presença de pesquisadores mulheres, geograficamente localizadas no estado de São Paulo. Apesar do grande número de artigos publicados por autores ligados a universidades, havia, também, aqueles ligados a centros não universitários. Com isso, observou-se uma contribuição a fim deque os princípios da Análise do Comportamento fossem discutidos em um contexto além das universidades.

Por fim, acredita-se que algumas limitações metodológicas desta pesquisa precisam ser destacadas. Apesar de certa pluralidade documental, a análise das publicações científicas ficou fortemente restrita ao periódico Modificação do Comportamento: Pesquisa e Aplicação. Além disso, não foi possível generalizar as observações advindas deste estudo para os variados grupos de analistas do comportamento, em atuação, no Brasil, à época. Como foram acessados documentos vinculados a uma associação, apenas, não houve como delimitar que os interesses de aplicação e referências tivessem sido os mesmos, para os diferentes analistas do comportamento atuantes, no país. Sugere-se, com isso, que novos estudos possam ampliar o corpus documental ora utilizado e, também, expandir os mecanismos de institucionalização da área, e.g., práticas de ensino, publicação de livros, entre outros.

Os dados tratados nesta pesquisa são de caráter documental. Toda a análise foi feita visando explorar um conjunto específico de fontes, portanto, permite conhecer apenas aqueles aspectos registrados nos documentos analisados. Deste modo, a caracterização proposta neste trabalho permitiu identificar personagens, instituições, publicações e temas de trabalhos organizados em torno das ações da AMC. Sabe-se que os dados aqui apresentados são parciais e que a história da institucionalização da Análise do Comportamento no Brasil inclui, ainda, outros personagens, instituições, publicações, temas, entre outros.

Todavia, os resultados desta pesquisa auxiliam em uma compreensão mais sofisticada da história da Análise do Comportamento, no Brasil, destacando elementos vinculados à institucionalização da 
área, por meio de sua aplicação. Nesse sentido, os dados apresentados neste artigo, contribuem com uma caracterização de publicações da área e, assim amplia o conhecimento de sua história e auxilia na identificação e preservação de sua memória.

\section{Referências}

Akera, A. (2017). Bringing radical behaviorism to revolutionary Brazil and back: Fred Keller's personalized system of instruction and cold war engineering education. Journal of the History of the Behavioral Sciences, 00, 1-19. doi: 10.1002/ jhbs.21871. Recuperado de https://www.ncbi. nlm.nih.gov/pubmed/28895137

Almeida, V. R. P. (1977). Redução da enurese noturna infantil: Considerações sobre os efeitos do procedimento de micções noturnas planejadas em intervalos de tempo gradualmente aumentados. Modificação de Comportamento: Pesquisa e Aplicação, (2), 31-60.

Associação de Modificação do Comportamento. (1975a). Estatutos da Associação de Modificação do Comportamento. Arquivos do Laboratório de Estudos Históricos em Análise do Comportamento da Pontifícia Universidade Católica de São Paulo (LeHac-PUC/SP).

Associação de Modificação do Comportamento. (1975b). Relação de sócios (até setembro de 75) da Associação de Modificação de Comportamento - São Paulo. Arquivos do Laboratório de Estudos Históricos em Análise do Comportamento da Pontifícia Universidade Católica de São Paulo (LeHac-PUC/SP).

Associação de Modificação do Comportamento. (1975c). AMC Boletim Informativo (Vol. 1, No. 2, maio). Arquivos do Laboratório de Estudos Históricos em Análise do Comportamento da Pontifícia Universidade Católica de São Paulo (LeHac-PUC/SP).

Associação de Modificação do Comportamento. (1975d). AMC Boletim Informativo (Vol. 3, No 4). Arquivos do Laboratório de Estudos Históricos em Análise do Comportamento da Pontifícia Universidade Católica de São Paulo (LeHac-PUC/SP).

Baer, D. M., Wolf, M. M., \& Risley, T. R. (1968).
Some current dimensions of Applied Behavior Analysis. Journal of Applied BehaviorAnalysis, 1, 91-97.doi: 10.1901/jaba.1968.1-91. Recuperado de https://www.ncbi.nlm.nih.gov/pmc/articles/ PMC1310980/

Botomé, S. P. (2006). Contribuições, participação, organização e representação da análise experimental do comportamento nos eventos e na organização da psicologia no Brasil: A ABPMC como condição e ponto de partida. Revista Brasileira de Terapia Comportamental e Cognitiva, 8(2), 217-231. doi: 10.31505/rbtcc. v8i2.103. Recuperado de http://www.usp.br/ rbtcc/index.php/RBTCC/article/view/103

Cândido, G. V. (2017a). Introdução da análise do comportamento no Brasil: A cadeira de psicologia de Rio Claro (1962-1963). Perspectivas em Análise do Comportamento, 8(1), 135-143. doi: 10.18761/pac.2016.027. Recuperado de https:// www.revistaperspectivas.org/perspectivas/article/view/225

Cândido, G. V. (2017b). Novas perspectivas para a história do sistema personalizado de ensino: Seus fundadores. Memorandum, 33, 51-67. Recuperado de https://periodicos.ufmg.br/index.php/memorandum/article/view/6651

Cândido, G. V., \& Massimi, M. (2016). Psicologia como ciência comportamental na Obra de Carolina Martuscelli Bori: Décadas de 1950 e 1960. Revista Argentina de Ciencias del Comportamiento, 8(2), p. 30-38. doi: 10.30882/1852.4206.v8.n2. Recuperado de https://revistas.unc.edu.ar/index.php/racc/article/view/10027

Cirino, S. D., Miranda, R. L., \& Cruz, R. N. (2012). The beginnings of behavior analysis laboratories in Brazil: A pedagogical view. History of Psychology, 15(3), 263-272. doi: 10.1037/ a0026306. Recuperado de https://www.ncbi. nlm.nih.gov/pubmed/23397916

Cirino, S. D., Miranda, R. L., \& Souza Júnior, E. J. (2012). The laboratory of experimental psychology: Establishing a psychological community at a Brazilian university. Interamerican Journal of Psychology, 46(1), 135-142. Recuperado de https://www.redalyc.org/articulo.oa?id=28424858013

Comitê de Publicações. (1977). Manual para 
preparação de originais. Modificação de Comportamento: Pesquisa e Aplicação, (2), 119-124.

Conselho Federal de Psicologia. (2013). Uma profissão de muitas e diferentes mulheres: resultado preliminar da pesquisa 2012. Brasília, DF: Autor.Recuperado de https://site.cfp.org.br/ wp-content/uploads/2013/03/Uma-profissaode-muitas-e-diferentes-mulheres-resultadopreliminar-da-pesquisa-2012.pdf

Costa, E. M. (1977). O uso de reforçamento diferencial no treinamento de professores: Um programa para instalar o comportamento de apresentação de conseqüências imediatas e explícitas ao aluno. Modificação de Comportamento: Pesquisa e Aplicação, (2), 91-103.

Cruz, R. N. (2006). História e historiografia da ciência: Considerações para pesquisa histórica em análise do comportamento. Revista Brasileira de Terapia Comportamental e Cognitiva, 8(2), 161178. doi: 10.31505/rbtcc.v8i2.98. Recuperado de http://www.usp.br/rbtcc/index.php/RBTCC/ article/view/98

Cury, S. (1996). Gary Martín e a experiência na PUC-SP. In R. A. Banaco (Org.), Sobre comportamento e cognição (Vol. 2, pp. 24-30). Santo André, SP: Arbytes.

Ferster, C. B. (1972). An experimental analysis of clinical phenomena. The Psychological Record, 22, 1-16.

Ferster, C. B., Culbertson, S., \& Perrott Boren, M. C. (1978). Princípios do comportamento. São Paulo, SP: HUCITEC.

Fleck, L. (2010). Gênese e desenvolvimento de um fato científico. Belo Horizonte, MG: Fabrefactum. (Obra original publicada em 1935).

Gonçalves, C. M. C. (1976). A pseudo-artificialidade de sistemas de vales. Modificação de Comportamento: Pesquisa e Aplicação, (1), 3340.

Gorayeb, R. (1991). História da Sociedade de Psicologia de Ribeirão Preto. Sociedade Brasileira de Psicologia. Recuperado de www. sbponline.org.br

Guedes, M. C., Cândido, G. V., Belloti, A. C., Giolo, J. C. C., Vieira, M. C., Matheus, N. M., \&
Gurgel, T. G. (2008). A introdução da análise do comportamento no Brasil: Vicissitudes. In M. A. Andery, N. Micheletto, \& T. M. Sério (Eds.), Behaviors: Ciência básica, ciência aplicada (Vol. 12, pp. 41-57). São Paulo, SP: Laboratório de Psicologia Experimental da Pontifícia Universidade Católica de São Paulo.

Guedes, M. C., Guimarães, T. A., \& Queiroz, A. B. (2007). A ABPMC e a institucionalização da análise do comportamento no Brasil. In M. A. Andery, N. Micheletto, \& T. M. Sério (Eds.), Behaviors: Ciência básica, ciência aplicada (Vol. 11, pp. 28-36). São Paulo, SP: Laboratório de Psicologia Experimental da Pontifícia Universidade Católica de São Paulo. Recuperado de http://abpmc.org.br/arquivos/ artigos/14653900912e1bb74f38.pdf

Guedes, M. C., Queiroz, A. B. M., Campos, A. C. H. F., Fonai, A. C. V., Silva, A. P. O., Sampaio, A. A. S., \& Pinto, V. J. C. (2006). Institucionalização da Análise do Comportamento no Brasil: Uma perspectiva histórica. In M. A. Andery, N. Micheletto, \& T. M. Sério (Eds.), Behaviors: Ciência básica, ciência aplicada (Vol. 10, pp. 17-29). São Paulo, SP: Laboratório de Psicologia Experimental da Pontifícia Universidade Católica de São Paulo. Recuperado de http://abpmc.org.br/arquivos/ artigos/1465390023eb916d08e.pdf

Guilhardi, H. J., Betini, M. E. S., \& Camargo, M. C. S. (1977). Aumento de freqüências de respostas acadêmicas para alterar a lentidão e eliminar comportamentos inadequados em um aluno de primeiro grau. Modificação de Comportamento: Pesquisa e Aplicação, (2), 1-30.

Hall, R. V. (1973). Manipulação de comportamento: Modificação do comportamento (Vol. 3). São Paulo, SP: EPU; Ed. Univ. S. Paulo.

Jabur, M. O. (1976). Efeitos do local de estudo no comportamento de estudar. Modificação de Comportamento: Pesquisa e Aplicação, (1), 1932.

Keller, F. S. (1988). Mulheres analistas do comportamento no Brasil (passado e presente). Psicologia: Teoria e Pesquisa, 4(1), 43-46. Recuperado de http://periodicos.unb.br/ojs248/index.php/revistaptp/article/view/20363

Knorr-Cetina, K. (1999). Epistemic cultures: How 
the sciences make knowledge. Cambridge, MA: Harvard University Press.

Lei no 4.119, de 27 de agosto de 1962. (1962, 17 de dezembro). Dispõe sobre os cursos de formação em Psicologia e regulamenta a profissão de psicólogo. Diário Oficial da União. Recuperado a partir de http://www.planalto.gov.br/civil_03/ leis/1950-1969/L4119.htm

Leite, S. A. S., Bastos, P. T. B., \& Bastos, N. S. C. (1976). Efeitos de um programa de matemática nos comportamentos da professora e dos alunos de uma classe de segundo ano do $1 .^{\circ}$ grau. Modificação de Comportamento: Pesquisa e Aplicação, (1), 1-18.

Leite, S. A. S., Leite, M. K. O. S., Ramires, V. L., Marcondes, Z. L. I., \& Guimarães, M. A. A. (1977). Efeitos de um programa remediativo de alfabetização nos comportamentos de crianças da $1 .^{\text {a }}$ série e da professora. Modificação de Comportamento: Pesquisa e Aplicação, (2), 61-76.

Leonardi, J. L. (2015). O lugar da terapia analítico-comportamental no cenário internacional das terapias comportamentais: Um panorama histórico. Perspectivas em Análise do Comportamento,6(2), 119-131.doi: 10.18761/ pac.2015.027. Recuperado de https://www. revistaperspectivas.org/perspectivas/article/ view/155/139

Madsen, C. H., Becker, W. C., \& Thomas, D. R. (1968). Rules, praise and ignoring: Elements of elementary classroom control. Journal of Applied Behavior Analysis, 1(2), 139-150. doi: 10.1901/jaba.1968.1-139. Recuperado de https://www.ncbi.nlm.nih.gov/pmc/articles/ PMC1310990/

Matos, M. A. (1998). Contingências para a análise comportamental no Brasil. Psicologia USP, 9(1), 1-6. doi: 10.1590/S010365641998000100014. Recuperado de http://www.revistas.usp.br/psicousp/article/view/107744

Mejias, N. P. (1976). A análise do comportamento aplicada à escola. Modificação de Comportamento: Pesquisa e Aplicação, (1), 4150.

Miranda, R. L. (2010). Laboratório de análise do comportamento no Brasil: Percursos na UFMG na década de 1970 (Dissertação de Mestrado).
Faculdade de Educação, Universidade Federal de Minas Gerais, Belo Horizonte, MG. Recuperado de http://www.bibliotecadigital. ufmg.br/dspace/bitstream/handle/1843/BUOS8E3KBS/dissertacao_rodrigo_miranda.pdf Modificação de Comportamento. (1974a, abril). Carta Informativa (No. 2). Arquivos do Laboratório de Estudos Históricos em Análise do Comportamento da Pontifícia Universidade Católica de São Paulo (LeHac-PUC/SP).

Modificação de Comportamento. (1974b, novembro). Carta Informativa (No. 5). Arquivos do Laboratório de Estudos Históricos em Análise do Comportamento da Pontifícia Universidade Católica de São Paulo (LeHac-PUC/SP).

Morris, E. K., Todd, J. T., Midgley, B. D., Schneider, S. M., \& Johnson, L. M. (1990). The history of behavior analysis: Some historiography and a bibliography. The Behavior Analyst, 13(2), 131158. Recuperado de https://www.ncbi.nlm.nih. gov/pmc/articles/PMC2733434/

Mota, A. M. G. F., Castro, E. A., \& Miranda, R. L. (2016). "Problemas de ajustamento" e "saúde mental": Controvérsias em torno de um objeto psicológico. In L. P. Almeida (Org.), Políticas públicas, cultura e produções sociais (Vol. 1, pp. 51-69). Campo Grande, MS: Editora da Universidade Católica Dom Bosco.

Mota, A. M. G. F., \& Miranda, R. L. (2017). Desvelando estilos de pensamento: "diagnósticos" nos Arquivos Brasileiros de Psicotécnica (1949-1968). In A. O. S. A. Duarte, M. F. P. Cassemiro, \& R. H. F. Campos. (Orgs.), Psicologia, educação e o debate ambiental: Questões históricas e contemporâneas (Vol. 1, pp. 277-288). Belo Horizonte, MG: FAE/UFMG; CDPHA.

Nale, N. (1998). Programação de ensino no Brasil: o papel de Carolina Bori. Psicologia USP, 9(1), 275-301.

Pereira, F. M., \& Pereira Neto, A. (2003). O psicólogo no Brasil: Notas sobre seu processo de profissionalização. Psicologia em Estudo, 8(2), 1927. Recuperado de http://www.scielo.br/pdf/pe/ v8n2/v8n2a02.pdf

Peron, S., Nogueira, E., Cândido, G. V., \& Massimi, M. (2015). Luiz Marcellino de Oliveira: Um protagonista da psicologia brasileira: Organização 
de acervo documental e análise histórica. Memorandum (Belo Horizonte), 29, 86-111. Recuperado de https://periodicos.ufmg.br/index.php/memorandum/article/view/6472

Queiroz, L. O., \& Guilhardi, H. J. (1980). Use of mediators in a behavior modification clinic in Brazil. In G. Martin, \&J. G. Osbourne, Helping in the community (pp. 259-271). New York: Plenun press.

Queiroz, L. O., Guilhardi, H. J., Martin, G. L., \& do Guedes, M. C. (1976). A university program in Brazil to develop psychologists with specialization in behavior modification. The Psychological Record, 26(2), 181-188. Recuperado de https:// link.springer.com/article/10.1007/BF03394376

Rosa, A., Huertas, J. A., \& Blanco, F. (1996). Metodología para la historia de la psicología. Madrid, Mad.: Alianza.

Rosas, P., Rosas, A., \& Xavier, I. B. (1988). Quantos e quem somos? In Conselho Federal de Psicologia (Org.), Quem é o psicólogo brasileiro? (pp.32-48). São Paulo: EDICON. Recuperado de http://newpsi.bvs-psi.org.br/ebooks2010/en/ Acervo_files/QuemPsicologoBrasileiro.pdf

Rubiano, M. R. B. (2005). Apresentando a Sociedade Brasileira de Psicologia. In M. A. Santos, L. L. M. Silva, \& C. P. Simon, Formação em psicologia: Serviços-escola em debate (pp. 83-116). São Paulo: Vetor.

Sabadini, A. A. Z. P. (2013). César Ades [Número Especial]. Psicologia: Ciência e Profissão, 33(spe), 4-13. ISSN 1414-9893. Recuperado em http://www.scielo.br/scielo.php?script=sci_ arttext\&pid=S1414-98932013000500002\&lng= en\&nrm=iso

Souza, F. M. S. (2011). Por uma história da análise do comportamento no Mato Grosso do Sul (Dissertação de Mestrado). Programa de Estudos Pós-graduados em Psicologia Experimental: Análise do Comportamento, Pontifícia Universidade Católica de São Paulo. Recuperado de https://tede2.pucsp.br/handle/ handle/ 16620

Souza Júnior, E. J., Miranda, R. L., \& Cirino, S. D. (2018). A recepção da instrução programada como abordagem da análise do comportamento no Brasil nos anos 1960 e 1970. História, Ciências, Saúde - Manguinhos, 25(2), 449- 467.doi: 10.1590/s0104-59702018000200009. Recuperado de http://www.scielo.br/scielo.php?script $=$ sci_arttext \&pid $=$ S010459702018000200449\&lng=en\&nrm=iso\&tlng $=\mathrm{pt}$

Todorov, J. C. (2006). Behavior analysis in Brazil. Avances en Psicología Latinoamericana, 24, 29 36.doi: 10.1590/S0102-37722010000500013. Recuperado de http://www.scielo. br/scielo.php?script=sci_arttext\&pid $=$ S0102-37722010000500013

Todorov, J. C., \& Hanna, E. S. (2010). Análise do comportamento no Brasil [Número Especial]. Psicologia: Teoria e Pesquisa, 26, 143-153. doi: 10.1590/S0102-37722010000500013. Recuperado de http://www.scielo.br/scielo.php?script $=$ sci_arttext \&pid=S010237722010000500013\&lng=en\&nrm=iso\&tlng $=\mathrm{pt}$

Tomanari, G. Y. (2005). Notícia: Maria Amélia Matos (1939-2005): Generosidade, competência, liderança. Psicologia: Teoria e Pesquisa, 21(2), 255-256. doi: 10.1590/S010237722005000200016. Recuperado de http:// www.scielo.br/scielo.php?script=sci_arttext\&p $\mathrm{id}=$ S0102-37722005000200016

Val, H. C. (2013). Conhecer e avançar: Um projeto de difusão da Análise do Comportamento em Minas Gerais (Dissertação de Mestrado). Programa de Estudos Pós-Graduados em Psicologia Experimental: Análise do Comportamento, Pontifícia Universidade Católica de São Paulo. Recuperado de https://tede2.pucsp.br/handle/ handle/16702

Witter, G. P. (2007). Importância das sociedades/ associações científicas: Desenvolvimento da ciência e formação do profissional-pesquisador. Boletim de Psicologia, 57, 1-14. Recuperado de http://pepsic.bvsalud. org/scielo.php?script=sci_arttext \&pid =S0006-59432007000100002

Wolf, M. M. (1993). Remembrances of issues past: Celebrating JABA's 25th anniversary. Journal of Applied Behavior Analysis, 26(4), 543-544. doi: 10.1901/jaba.1993.26-543. Recuperado de https://www.ncbi.nlm.nih.gov/pmc/articles/ PMC1297886/ 
Zibas, D. M. L. (1977). Modificação de comportamento de inibição (ou de isolamento) de criança em idade escolar - estudo de um caso. Modificação de Comportamento: Pesquisa e Aplicação, (2), 77-90.

\section{Informações do Artigo}

Histórico do artigo:

Recebido em: 27/03/2019

Tramitado em: 19/06/2020

Primeira decisão editorial: 06/07/2020

Aceito em: 22/07/2020

Editora Associada: Carolina Laurenti 